\title{
The Distance Modulus in Dark Energy and Cardassian Cosmologies via the Hypergeometric Function
}

\author{
Lorenzo Zaninetti \\ Physics Department, via P. Giuria 1, Turin, Italy \\ Email: zaninetti@ph.unito.it
}

How to cite this paper: Zaninetti, L. (2019) The Distance Modulus in Dark Energy and Cardassian Cosmologies via the Hypergeometric Function. International Journal of Astronomy and Astrophysics, 9, 231-246.

https://doi.org/10.4236/ijaa.2019.93017

Received: June 11, 2019

Accepted: September 1, 2019

Published: September 4, 2019

Copyright $\odot 2019$ by author(s) and Scientific Research Publishing Inc. This work is licensed under the Creative Commons Attribution International License (CC BY 4.0).

http://creativecommons.org/licenses/by/4.0/

\begin{abstract}
The presence of the dark energy allows both the acceleration and the expansion of the universe. In the case of a constant equation of state for dark energy we derived an analytical solution for the Hubble radius in terms of the hypergeometric function. An approximate Taylor expansion of order seven is derived for both the constant and the variable equation of state for dark energy. In the case of the Cardassian cosmology, we also derived an analytical solution for the Hubble radius in terms of the hypergeometric function. The astronomical samples of the distance modulus for Supernova (SN) of type Ia allows the derivation of the involved cosmological in the case of constant equation of state, variable equation of state and Cardassian cosmology.
\end{abstract}

\section{Keywords}

Cosmology, Observational Cosmology, Distances, Redshifts, Radial Velocities, Spatial Distribution of Galaxies, Magnitudes and Colors, Luminosities

\section{Introduction}

The name dark energy started to be used by [1] in order to explain both the expansion and both the acceleration of the universe. In a few years the dark energy was widely used as a cosmological model to be tested. Many review papers have been written; we select among others a general review by [2] and a theoretical review by [3]. The term wCDM has been introduced to classify the case of constant equation of state and we will use in the following wzCDM to classify the variable equation of state. The Cardassian cosmology started with [4] and was introduced in order to model both the expansion and the acceleration of 
the universe, the name from a humanoid race in Star Trek. As an example [5] derived the cosmological parameters for the original Cardassian expansion and the modified polytropic Cardassian expansion. The cosmological theories can be tested on the samples of Supernova (SN) of type Ia. The first sample to be used to derive the cosmological parameters contained $7 \mathrm{SNs}$, see [6], the second one contained 34 SNs, see [7] and the third one contained 42 SNs, see [8]. The above historical samples allowed to derive the cosmological parameters for the expanding and accelerating universe. At the moment of writing the astronomical research is focused on value of the distance modulus versus the redshift: the Union 2.1 compilation contains $580 \mathrm{SNs}$, see [9], and the joint light-curve analysis (JLA) contains $740 \mathrm{SNs}$, see [10]. The above observations can be done up to a limited value in redshift $z \approx 1.7$, we, therefore, speak of evaluation of the distance modulus at low redshift. This limited range can be extended up $z \approx 8$, the high redshift region, analyzing the Gamma-Ray Burst (GRB) and, as an example, [11] has derived the distance modulus for 59 calibrated highredshift GRBs, the so-called "Hymnium" GRBs sample. This paper reviews in Section 2.1. The $\Lambda \mathrm{CDM}$ cosmology evaluates the basic integral of $\mathrm{wCDM}$ cosmology in Section 3, introduces a Taylor expansion for the basic integral of wzCDM cosmology in Section 4 and analyzes the Cardassian model in Section 5. The parameters which characterize the three cosmologies are derived via the Levenberg-Marquardt method in Section 6.

\section{Preliminaries}

This section reviews the $\Lambda \mathrm{CDM}$ cosmology and the adopted statistics.

\subsection{The Standard Cosmology}

In $\Lambda \mathrm{CDM}$ cosmology the Hubble distance $D_{\mathrm{H}}$ is defined as

$$
D_{\mathrm{H}} \equiv \frac{c}{H_{0}} \text {. }
$$

The first parameter is $\Omega_{\mathrm{M}}$

$$
\Omega_{\mathrm{M}}=\frac{8 \pi G \rho_{0}}{3 H_{0}^{2}},
$$

where $G$ is the Newtonian gravitational constant, $H_{0}$ is the Hubble constant and $\rho_{0}$ is the mass density at the present time. The second parameter is $\Omega_{\Lambda}$

$$
\Omega_{\Lambda} \equiv \frac{\Lambda c^{2}}{3 H_{0}^{2}},
$$

where $\Lambda$ is the cosmological constant, see [12]. These two parameters are connected with the curvature $\Omega_{K}$ by

$$
\Omega_{\mathrm{M}}+\Omega_{\Lambda}+\Omega_{K}=1 \text {. }
$$

The comoving distance, $D_{\mathrm{C}}$ is

$$
D_{\mathrm{C}}=D_{\mathrm{H}} \int_{0}^{z} \frac{\mathrm{d} z^{\prime}}{E\left(z^{\prime}\right)}
$$


where $E(z)$ is the "Hubble function"

$$
E(z)=\sqrt{\Omega_{\mathrm{M}}(1+z)^{3}+\Omega_{K}(1+z)^{2}+\Omega_{\Lambda}} .
$$

In the case of $\Omega_{K}$, we have the flat case.

\subsection{The Statistics}

The adopted statistical parameters are the percent error, $\delta$, between theoretical value and approximated value, the merit function $\chi^{2}$ evaluated as

$$
\chi^{2}=\sum_{i=1}^{N}\left[\frac{y_{i, \text { theo }}-y_{i, o b s}}{\sigma_{i}}\right]^{2}
$$

where $y_{i, o b s}$ and $\sigma_{i}$ represent the observed value and its error at position $i$ and $y_{i, \text { theo }}$ the theoretical value at position $i$, the reduced merit function $\chi_{\text {red }}^{2}$, the Akaike information criterion (AIC), the number of degrees of freedom $N F=n-k$ where $n$ is the number of bins and $k$ is the number of parameters and the goodness of the fit as expressed by the probability $Q$.

\section{Constant Equation of State}

In dark matter cosmology, $\mathrm{wCDM}$, the Hubble radius is

$$
d_{H}\left(z ; \Omega_{\mathrm{M}}, w, \Omega_{D E}\right)=\frac{1}{\sqrt{(1+z)^{3} \Omega_{\mathrm{M}}+\Omega_{D E}(1+z)^{3+3 w}}},
$$

where $w$ parametrizes the dark energy and is constant, see Equation (3.4) in [13] or Equation (18) in [14] for the luminosity distance.

In flat cosmology

$$
\Omega_{\mathrm{M}}+\Omega_{D E}=1
$$

and the Hubble radius becomes

$$
d_{H}\left(z ; \Omega_{\mathrm{M}}, w\right)=\frac{1}{\sqrt{(1+z)^{3} \Omega_{\mathrm{M}}+\left(1-\Omega_{\mathrm{M}}\right)(1+z)^{3+3 w}}} .
$$

The indefinite integral in the variable $z$ of the above Hubble radius, $I z$, is

$$
I z\left(z ; \Omega_{\mathrm{M}}, w\right)=\int d_{H}\left(z ; \Omega_{\mathrm{M}}, w\right) \mathrm{d} z .
$$

\subsection{The Analytical Solution}

In order to solve the indefinite integral we perform a change of variable $1+z=t^{1 / 3}$

$$
I z\left(t ; \Omega_{\mathrm{M}}, w\right)=\frac{1}{3} \int \frac{1}{\sqrt{-t\left(\left(-1+\Omega_{\mathrm{M}}\right) t^{w}-\Omega_{\mathrm{M}}\right)} t^{2 / 3}} \mathrm{~d} t .
$$

The indefinite integral is

$$
I z\left(t ; \Omega_{\mathrm{M}}, w\right)=\frac{-2{ }_{2} \mathrm{~F}_{1}\left(\frac{1}{2},-\frac{1}{6} w^{-1} ; 1-\frac{1}{6} w^{-1} ;-\frac{t^{w}-\left(1-\Omega_{\mathrm{M}}\right)}{\Omega_{\mathrm{M}}}\right)}{\sqrt{\Omega_{\mathrm{M}}} \sqrt[6]{t}},
$$


where ${ }_{2} \mathrm{~F}_{1}(a, b ; c ; z)$ is the regularized hypergeometric function, see Appendix B. This dependence of the above integral upon the hypergeometric function has been recognized but not developed by [15].

We now return to the variable $z$, the redshift, and the indefinite integral becomes

$$
I z\left(z ; \Omega_{\mathrm{M}}, w\right)=\frac{-2{ }_{2} \mathrm{~F}_{1}\left(\frac{1}{2},-\frac{1}{6} w^{-1} ; 1-\frac{1}{6} w^{-1} ;-\frac{\left(-z^{3}+3 z^{2}+3 z+1\right)^{w}\left(1-\Omega_{\mathrm{M}}\right)}{-\Omega_{\mathrm{M}}}\right)}{\sqrt{\Omega_{\mathrm{M}}} \sqrt[6]{z^{3}+3 z^{2}+3 z+1}} .
$$

We denote by $F\left(z ; \Omega_{\mathrm{M}}, w\right)$ the definite integral

$$
F\left(z ; \Omega_{\mathrm{M}}, w\right)=I z\left(z=z ; \Omega_{\mathrm{M}}, w\right)-I z\left(z=0 ; \Omega_{\mathrm{M}}, w\right) .
$$

\subsection{The Taylor Expansion}

We evaluate the integrand of the integral (11) with a first series expansion, $T_{I}$ about $z=0$, denoted by $I$ and a second series expansion, $T_{I I}$, about $z=1$, denoted by $I I$. The order of expansion for the two series is 7 . The integration of $T_{I}$ in $\mathrm{z}$ is denoted by $I z_{I, 7}$ and gives

$$
I z_{I, 7}\left(z ; \Omega_{\mathrm{M}}, w\right)=\sum_{i=1}^{i=7} c_{I, i} z^{i}
$$

and the coefficients, $c_{I, i}$, are reported in Appendix A. The integral, $I z_{I I, 7}$ of the second Taylor expansion about $z=1, T_{I I}$ is complicated and we limit ourselves to order 2, $I z_{I I, 2}$, see Appendix A. The two definite integrals, $F_{I, 7}\left(z ; \Omega_{\mathrm{M}}, w\right)$ and $F_{I I, 7}\left(z ; \Omega_{\mathrm{M}}, w\right)$ are

$$
F_{I, 7}\left(z ; \Omega_{\mathrm{M}}, w\right)=I z_{I, 7}\left(z=z ; \Omega_{\mathrm{M}}, w\right)-I z_{I, 7}\left(z=0 ; \Omega_{\mathrm{M}}, w\right),
$$

and

$$
F_{I I, 7}\left(z ; \Omega_{\mathrm{M}}, w\right)=I z_{I I, 7}\left(z=z ; \Omega_{\mathrm{M}}, w\right)-I z_{I I, 7}\left(z=0 ; \Omega_{\mathrm{M}}, w\right) .
$$

The percent error, $\delta$, between the analytical integral $F$ and the two approximations, $F_{I, 7}$ and $F_{I I, 7}$ is evaluated as

$$
\begin{gathered}
\delta_{I}=\left|1-\frac{F_{I, 7}}{F}\right| \times 100 \\
\delta_{I I}=\left|1-\frac{F_{I I, 7}}{F}\right| \times 100 .
\end{gathered}
$$

On inserting the astrophysical parameters as reported in Table 1 we have $\delta_{I}=\delta_{I I}$ at $z \approx 0.58$, see Figure 1.

The above value in $\mathrm{z}$ will, therefore, be the boundary between region I and region II for the Taylor approximation of the definite integral

$$
F_{7}\left(z ; \Omega_{\mathrm{M}}, w\right)= \begin{cases}F_{I I, 7}\left(z ; \Omega_{\mathrm{M}}, w\right), & 0.58 \leq z \leq 1.4 \\ F_{I, 7}\left(z ; \Omega_{\mathrm{M}}, w\right), & 0<z<0.58\end{cases}
$$


Table 1. Numerical values from the Union 2.1 compilation of $\chi^{2}, \chi_{\text {red }}^{2}$ and $Q$, where $k$ stands for the number of parameters.

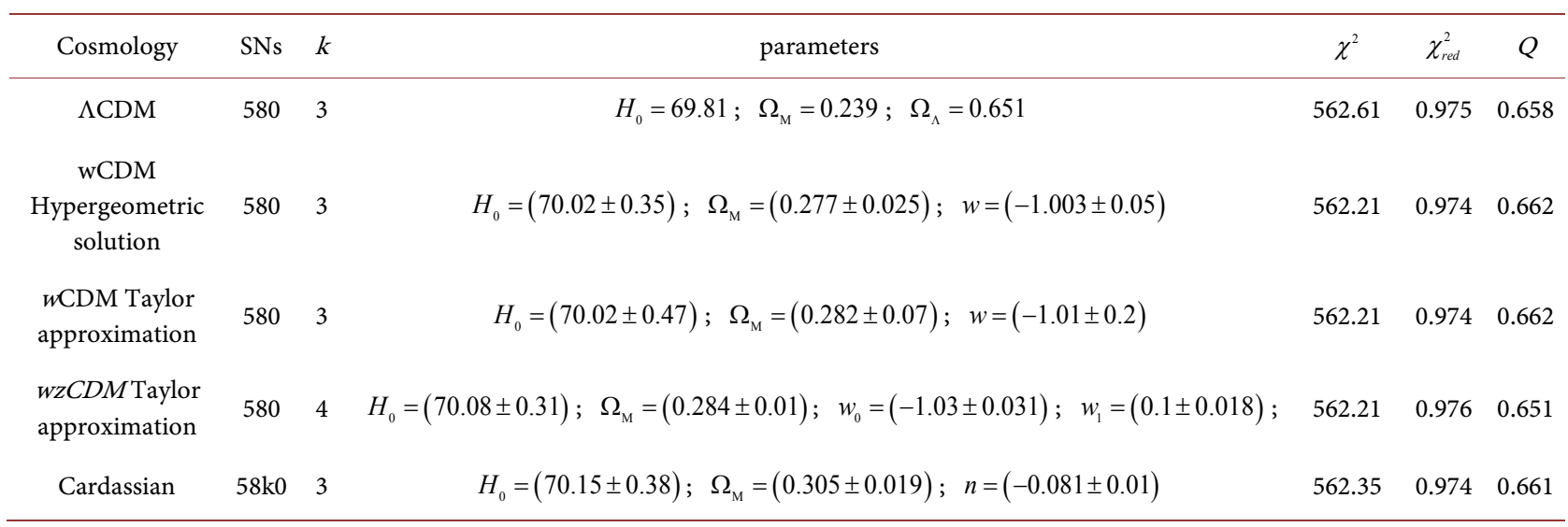

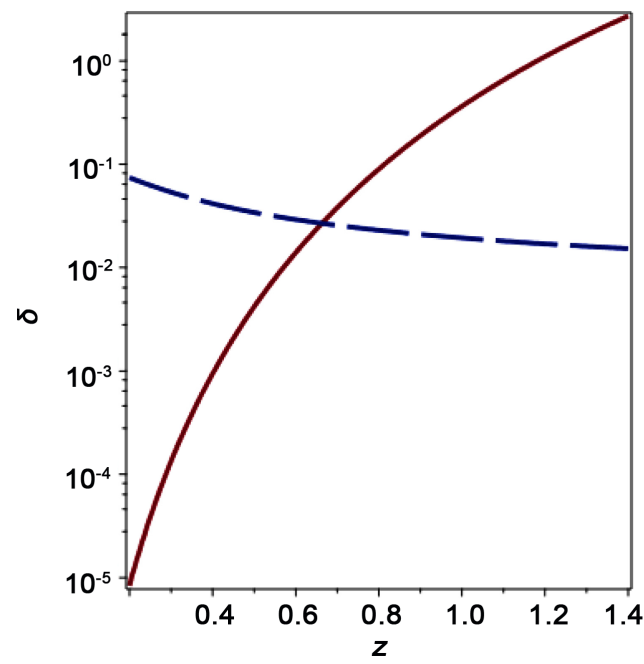

Figure 1. Numerical values of $\delta_{I}$ (full red line) and $\delta_{I I}$ (dashed blue line) as function of the redshift, parameters as in Table 1 .

\section{Variable Equation of State}

The dark energy as function of the redshift is assumed to be

$$
w(z)=w_{0}+w_{1} \frac{z}{1+z},
$$

where $w_{0}$ and $w_{1}$ are two parameters to be fixed by the fit. The Hubble radius in $w Z C D M$ cosmology is

$$
d_{H}\left(z ; \Omega_{\mathrm{M}}, w_{0}, w_{1}\right)=\frac{1}{\sqrt{(1+z)^{3} \Omega_{\mathrm{M}}+\left(1-\Omega_{\mathrm{M}}\right)(1+z)^{3 w_{0}+3 w_{1}+3} \mathrm{e}^{-3 \frac{w_{1} z}{1+z}}}}
$$

which is the same as Equation (20) in [14]. The above integral does not yet have an analytical expression and we evaluate the integrand with a first series expansion about $z=0$ and a second series expansion about $z=1$. Also here the order of the two series expansion is 7 . The integration in $z$ is denoted by $I w z_{I, 7}$ and gives 


$$
I w z_{I, 7}\left(z ; \Omega_{\mathrm{M}}, w_{0}, w_{1}\right)=\sum_{i=1}^{i=7} c_{I, i} z^{i}
$$

and the first five coefficients, $c_{I, i}$, are reported in Appendix C. The integral, $I w z_{I I, 7}$ of the second Taylor expansion about $z=1$ is complicated and we limit ourselves to order 2, Iwz $z_{I I, 2}$, see Appendix C. The two definite integrals, $F w z_{I, 7}\left(z ; \Omega_{\mathrm{M}}, w_{0}, w_{1}\right)$ and $F w z_{I I, 7}\left(z ; \Omega_{\mathrm{M}}, w_{0}, w_{1}\right)$ are

$F w z_{I, 7}\left(z ; \Omega_{\mathrm{M}}, w_{0}, w_{1}\right)=I w z_{I, 7}\left(z=z ; \Omega_{\mathrm{M}}, w_{0}, w_{1}\right)-I w z_{I, 7}\left(z=0 ; \Omega_{\mathrm{M}}, w_{0}, w_{1}\right)$,

and

$$
F w z_{I I, 7}\left(z ; \Omega_{\mathrm{M}}, w_{0}, w_{1}\right)=I w z_{I I, 7}\left(z=z ; \Omega_{\mathrm{M}}, w_{0}, w_{1}\right)-I w z_{I I, 7}\left(z=0 ; \Omega_{\mathrm{M}}, w_{0}, w_{1}\right) .(26
$$

Finally the definite integral, $F w z$, is

$$
F w z_{7}\left(z ; \Omega_{\mathrm{M}}, w_{0}, w_{1}\right)= \begin{cases}F w z_{I I, 7}\left(z ; \Omega_{\mathrm{M}}, w_{0}, w_{1}\right), & 0.58 \leq z \leq 1.4 \\ F w z_{I, 7}\left(z ; \Omega_{\mathrm{M}}, w_{0}, w_{1}\right), & 0<z<0.58\end{cases}
$$

The above definite integral can also be evaluated in a numerical way,

$$
F w z_{\text {num }}\left(z ; \Omega_{\mathrm{M}}, w_{0}, w_{1}\right) \text {. }
$$

\section{Cardassian Cosmology}

In flat Cardassian cosmology the Hubble radius is

$$
d_{H}\left(z ; \Omega_{\mathrm{M}}, w, n\right)=\frac{1}{\sqrt{(1+z)^{3} \Omega_{\mathrm{M}}+\left(1-\Omega_{\mathrm{M}}\right)(1+z)^{3 n}}},
$$

where $n$ is a variable parameter, $n=0$ means $\Lambda$ CDM cosmology, see Equation (17) in [14]. The indefinite integral in the variable $z$ of the above Hubble radius, $I z$, is

$$
I z\left(z ; \Omega_{\mathrm{M}}, n\right)=\int d_{H}\left(z ; \Omega_{\mathrm{M}}, n\right) \mathrm{d} z .
$$

Also here in order to solve the indefinite integral we perform a change of variable $1+z=t^{1 / 3}$

$$
I z\left(t ; \Omega_{\mathrm{M}}, n\right)=\frac{1}{3} \int \frac{1}{\sqrt{-t^{n} \Omega_{\mathrm{M}}+\Omega_{\mathrm{M}} t+t^{n}} t^{2 / 3}} \mathrm{~d} t .
$$

The indefinite integral is

$$
I z\left(t ; \Omega_{\mathrm{M}}, n\right)=\frac{-2{ }_{2} \mathrm{~F}_{1}\left(1 / 2,-(6 n-6)^{-1} ; \frac{6 n-7}{6 n-6} ; \frac{t^{n-1}\left(\Omega_{\mathrm{M}}-1\right)}{\Omega_{\mathrm{M}}}\right)}{\sqrt{\Omega_{\mathrm{M}}} \sqrt[6]{t}},
$$

where ${ }_{2} \mathrm{~F}_{1}(a, b ; c ; z)$ is the regularized hypergeometric function. We now return to the original variable $z$ as function of $z$ which is

$$
I z\left(z ; \Omega_{\mathrm{M}}, n\right)=\frac{-2{ }_{2} \mathrm{~F}_{1}\left(1 / 2,-(6 n-6)^{-1} ; \frac{6 n-7}{6 n-6} ; \frac{\left((1+z)^{3}\right)^{n-1}\left(\Omega_{\mathrm{M}}-1\right)}{\Omega_{\mathrm{M}}}\right)}{\sqrt{\Omega_{\mathrm{M}}} \sqrt[6]{(1+z)^{3}}} .
$$


We denote by $F_{c}\left(z ; \Omega_{\mathrm{M}}, n\right)$ the definite integral

$$
F_{c}\left(z ; \Omega_{\mathrm{M}}, n\right)=I z\left(z=z ; \Omega_{\mathrm{M}}, n\right)-I z\left(z=0 ; \Omega_{\mathrm{M}}, n\right) .
$$

\section{The Distance Modulus}

The luminosity distance, $d_{\mathrm{L}}$, for wCDM cosmology in the case of the analytical solution is

$$
d_{\mathrm{L}}\left(z ; c, H_{0}, \Omega_{\mathrm{M}}, w\right)=\frac{c}{H_{0}}(1+z) F\left(z ; \Omega_{\mathrm{M}}, w\right),
$$

where $F\left(z ; \Omega_{\mathrm{M}}, w\right)$ is given by Equation (15) and in the case of the Taylor approximation is

$$
d_{\mathrm{L}, 7}\left(z ; c, H_{0}, \Omega_{\mathrm{M}}, w\right)=\frac{c}{H_{0}}(1+z) F_{7}\left(z ; \Omega_{\mathrm{M}}, w\right),
$$

where $F_{7}\left(z ; \Omega_{\mathrm{M}}, w\right)$ is given by Equation (21). The distance modulus in the case of the analytical solution for wCDM is

$$
(m-M)=25+5 \log _{10}\left(d_{\mathrm{L}}\left(z ; c, H_{0}, \Omega_{\mathrm{M}}, w\right)\right),
$$

and in the case of the Taylor approximation

$$
(m-M)_{7}=25+5 \log _{10}\left(d_{\mathrm{L}, 7}\left(z ; c, H_{0}, \Omega_{\mathrm{M}}, w\right)\right) .
$$

In the case of variable equation of state, $w z C D M$, the numerical luminosity distance is

$$
d_{\mathrm{L}, \text { num }}\left(z ; c, H_{0}, \Omega_{\mathrm{M}}, w_{0}, w_{1}\right)=\frac{c}{H_{0}}(1+z) F w z_{\text {num }}\left(z ; \Omega_{\mathrm{M}}, w_{0}, w_{1}\right),
$$

where $F w z_{\text {mum }}\left(z ; \Omega_{\mathrm{M}}, w_{0}, w_{1}\right)$ is the definite numerical integral and the Taylor approximation for the luminosity distance is

$$
d_{\mathrm{L}, 7}\left(z ; c, H_{0}, \Omega_{\mathrm{M}}, w_{0}, w_{1}\right)=\frac{c}{H_{0}}(1+z) F w z_{7}\left(z ; \Omega_{\mathrm{M}}, w_{0}, w_{1}\right),
$$

where $F w z_{7}\left(z ; \Omega_{\mathrm{M}}, w_{0}, w_{1}\right)$ is given by Equation (27). In $w z C D M$, the numerical distance modulus is

$$
(m-M)_{\text {num }}=25+5 \log _{10}\left(d_{\mathrm{L}, \text { num }}\left(z ; c, H_{0}, \Omega_{\mathrm{M}}, w_{0}, w_{1}\right)\right),
$$

and the Taylor approximated distance modulus is

$$
(m-M)_{7}=25+5 \log _{10}\left(d_{\mathrm{L}, 7}\left(z ; c, H_{0}, \Omega_{\mathrm{M}}, w_{0}, w_{1}\right)\right) .
$$

In the case of Cardassian cosmology the luminosity distance is

$$
d_{\mathrm{L}}\left(z ; c, H_{0}, \Omega_{\mathrm{M}}, n\right)=\frac{c}{H_{0}}(1+z) F_{c}\left(z ; \Omega_{\mathrm{M}}, n\right),
$$

where $F_{c}\left(z ; \Omega_{\mathrm{M}}, n\right)$ is given by Equation (33) and the distance modulus is

$$
(m-M)=25+5 \log _{10}\left(d_{\mathrm{L}}\left(z ; c, H_{0}, \Omega_{\mathrm{M}}, n\right)\right) .
$$

The cosmological parameters unknown are three, $H_{0}, \Omega_{\mathrm{M}}$ and $w$, in the case of $\mathrm{wCDM}$ and four, $H_{0}, \Omega_{\mathrm{M}}, w_{0}$ and $w_{1}$, in the case of $w z C D M$. In flat 
Cardassian cosmology the number of parameters is three, $H_{0}, \Omega_{\mathrm{M}}$ and $n$. In the presence of a given sample for the distance modulus, we can map the chi-square as given by Formula (7), see Figure 2 in the case of wCDM with hypergeometric solution. The above cosmological parameters are obtained by a fit of the astronomical data for the distance modulus of $\mathrm{SNs}$ via the Levenberg-Marquardt method (subroutine MRQMIN in [16]) which minimizes the chi-square as given by Formula (7). Table 1 presents the above cosmological parameters for the Union 2.1 compilation of SNs and Figure 3 reports the best fit. As a practical example of the utility of the cosmological parameters determination, we report the distance modulus in an explicit form for the Union 2.1 compilation in wCDM.

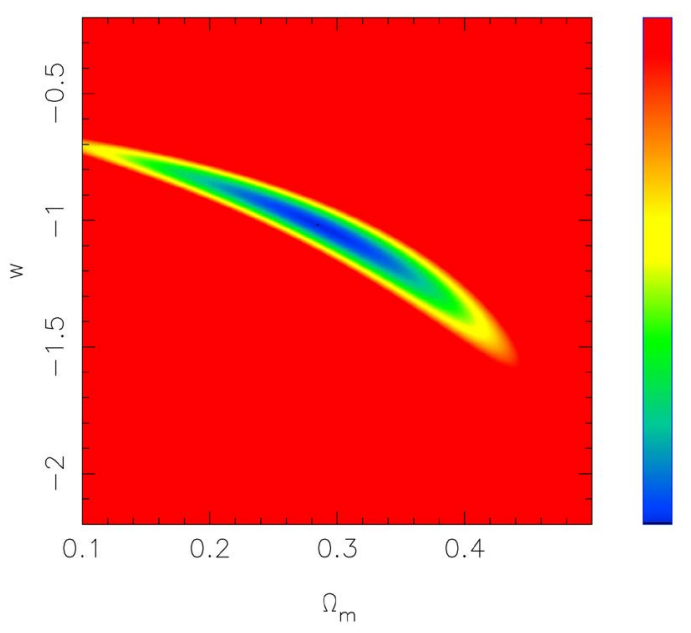

Figure 2. Map of the $\chi^{2}$ in wCDM cosmology when $H_{0}=(70.02 \pm 0.35)$.

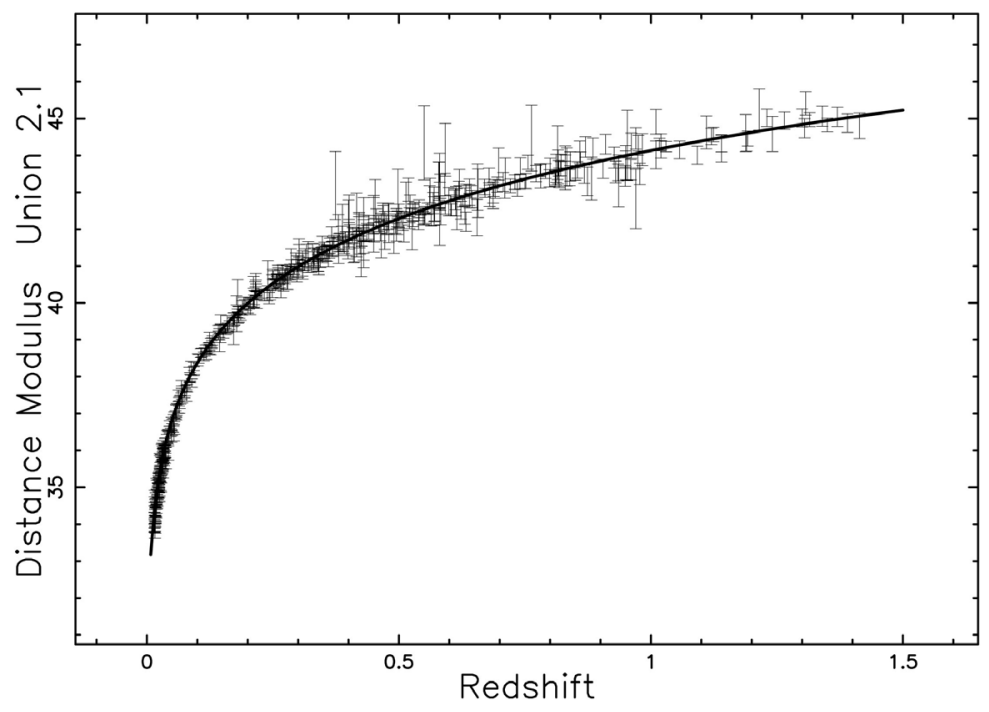

Figure 3. Hubble diagram for the Union 2.1 compilation. The solid line represents the best fit for the exact distance modulus in wCDM cosmology as represented by Equation (36). Parameters as in third line of Table 1; Union 2.1 compilation. 


$$
\begin{aligned}
& (m-M)=5+5 \frac{1}{\ln (10)} \times \ln (4281.52(1+z) \\
& \left.\times\left(-3.8 \frac{{ }_{2} \mathrm{~F}_{1}\left(0.1661, \frac{1}{2} ; 1.1661 ;-2.6101\left(z^{3}+3 z^{2}+3 z+1\right)^{-1.003}\right)}{\sqrt[6]{z^{3}+3 z^{2}+3 z+1}}+3.4146\right)\right)
\end{aligned}
$$

when $0<z<1.4$,

And in flat Cardassian cosmology

$$
\begin{aligned}
& (m-M)=\frac{1}{\ln (10)} 25 \ln (10) \\
& +5 \ln \left(-4273.59(1+z)\left(3.62142\left(z^{3}+3 z^{2}+3 z+1\right)^{-0.16666}\right.\right. \\
& \left.\left.\times{ }_{2} \mathrm{~F}_{1}\left(0.15417,1 / 2 ; 1.1541 ;-2.2786\left(z^{3}+3 z^{2}+3 z+1\right)^{-1.081}\right)-3.304\right)\right)
\end{aligned}
$$

when $0<z<1.4$.

Table 2 reports the cosmological parameters for the JLA compilation and Figure 4 the connected fit.

The presence of the "Hymnium" GRBs sample allows to calibrate the distance modulus in the high redshift region (see Table 3 and Figure 5).

The extension of the Hubble diagram to the GRBs, as an example, has been implemented in [11] [17] [18] [19] [20].

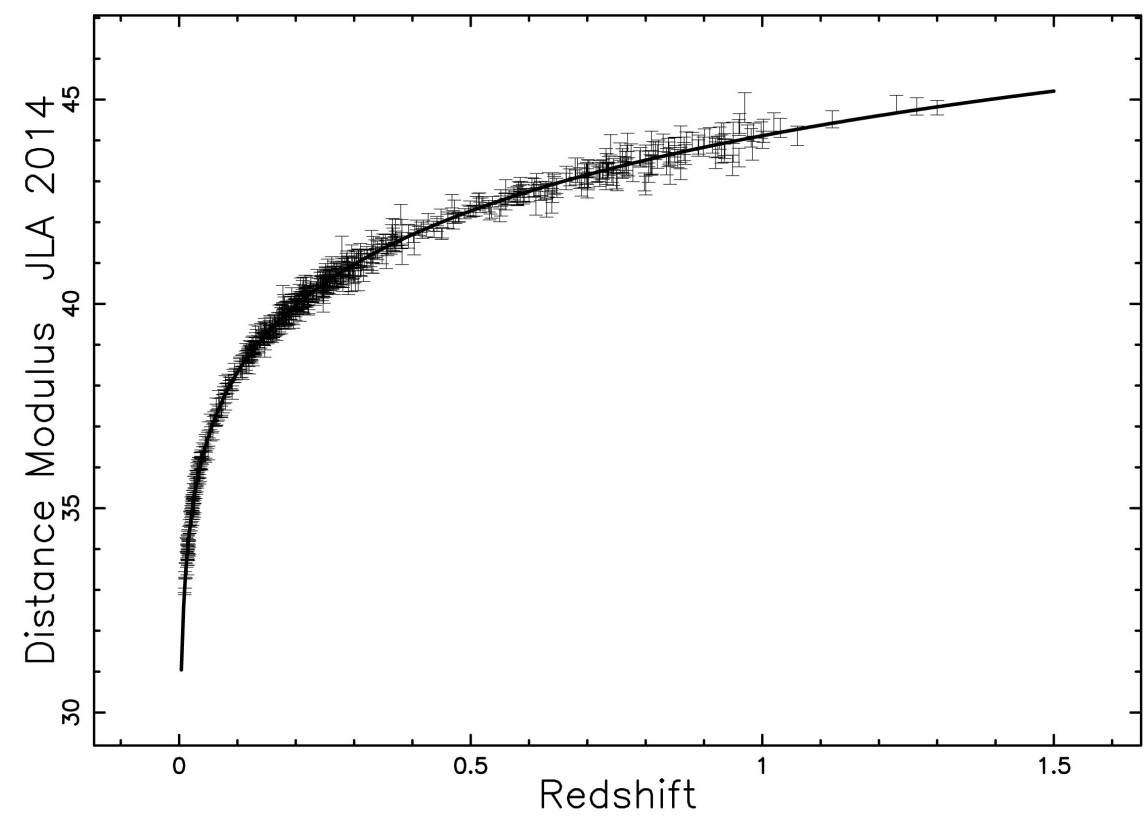

Figure 4. Hubble diagram for the JLA compilation. The solid line represents the best fit for the exact distance modulus in WCDM cosmology as represented by Equation (36). Parameters as in the third line of Table 2; JLA compilation. 


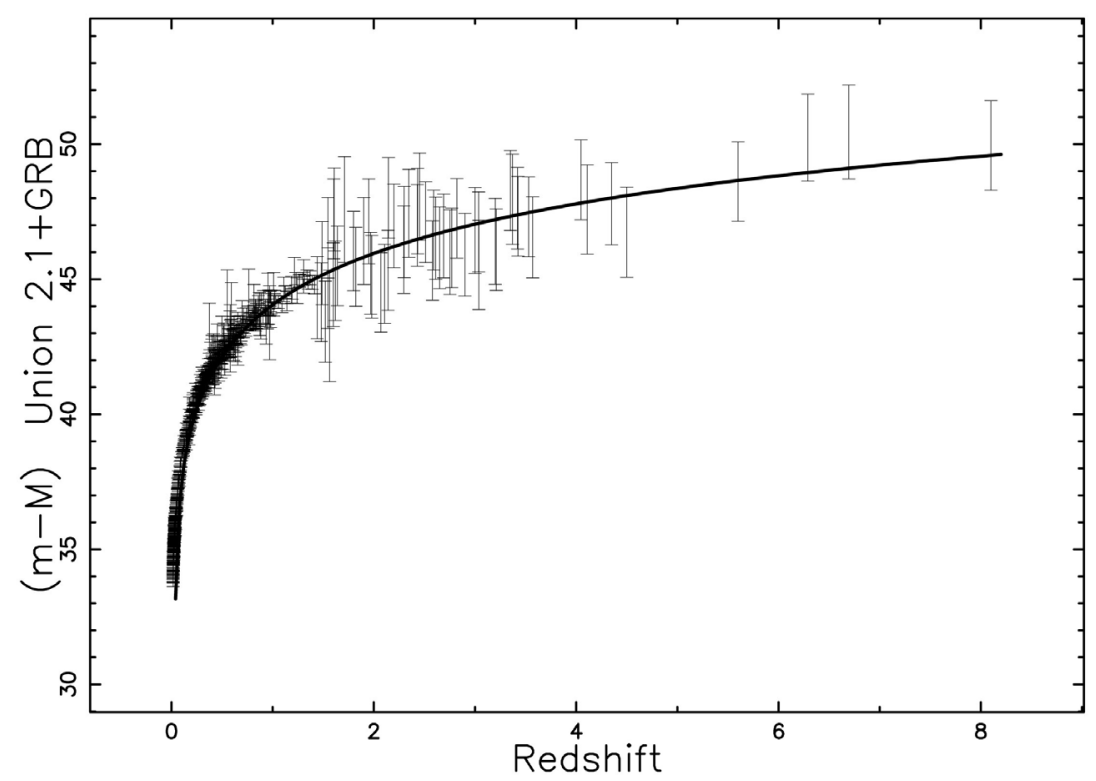

Figure 5. Hubble diagram for the Union 2.1 compilation + the "Hymnium" GRBs sample. The solid line represents the best fit for the exact distance modulus in wCDM cosmology as represented by Equation (36). Parameters as in second line of Table 3.

Table 2. Numerical values for the JLA compilation of $\chi^{2}, \chi_{\text {red }}^{2}$ and $Q$, where $k$ stands for the number of parameters.

\begin{tabular}{|c|c|c|c|c|c|c|}
\hline Cosmology & SNs & $k$ & parameters & $\chi^{2}$ & $\chi_{\text {red }}^{2}$ & $Q$ \\
\hline$\Lambda \mathrm{CDM}$ & 740 & 3 & $H_{0}=69.39 ; \Omega_{\mathrm{M}}=0.18 ; \Omega_{\Lambda}=0.537$ & 625.74 & 0.849 & 0.99 \\
\hline $\begin{array}{l}\text { wCDM } \\
\text { Hypergeometric } \\
\text { solution }\end{array}$ & 740 & 3 & $H_{0}=(69.71 \pm 0.5) ; \quad \Omega_{\mathrm{M}}=(0.293 \pm 0.021) ; \quad w=(-0.996 \pm 0.08)$ & 627.908 & 0.851 & 0.998 \\
\hline $\begin{array}{l}\text { wCDM Taylor } \\
\text { approximation }\end{array}$ & 740 & 4 & $H_{0}=(69.99 \pm 0.29) ; \quad \Omega_{\mathrm{M}}=(0.133 \pm 0.13) ; w=(-0.709 \pm 0.18)$ & 625.69 & 0.848 & 0.998 \\
\hline $\begin{array}{l}\text { wzCDM Taylor } \\
\text { approximation }\end{array}$ & 740 & 4 & $H_{0}=(69.99 \pm 0.29) ; \quad \Omega_{\mathrm{M}}=(0.3 \pm 0.009) ; \quad w_{0}=(-1.05 \pm 0.027) ; w_{1}=(0.097 \pm 0.01) ;$ & 628.76 & 0.854 & 0.998 \\
\hline Cardassian & 740 & 3 & $H_{0}=(70.036 \pm 0.44) ; \quad \Omega_{\mathrm{M}}=(0.301 \pm 0.019) ; \quad n=(-0.055 \pm 0.0045)$ & 628.73 & 0.863 & 0.999 \\
\hline
\end{tabular}

Table 3. Numerical values from the Union 2.1 compilation + the "Hymnium" GRBs sample of $\chi^{2}, \chi_{\text {red }}^{2}$ and $Q$, where $k$ stands for the number of parameters.

\begin{tabular}{|c|c|c|c|c|c|c|}
\hline Cosmology & SNs & $k$ & parameters & $\chi^{2}$ & $\chi_{\text {red }}^{2}$ & $Q$ \\
\hline$\Lambda \mathrm{CDM}$ & 639 & 3 & $H_{0}=69.80 ; \Omega_{\mathrm{M}}=0.239 ; \Omega_{\Lambda}=0.651$ & 586.08 & 0.921 & 0.922 \\
\hline $\begin{array}{c}\text { wCDM } \\
\text { Hypergeometric } \\
\text { solution }\end{array}$ & 639 & 3 & $H_{0}=(70.12 \pm 0.4) ; \quad \Omega_{\mathrm{M}}=(0.294 \pm 0.024) ; \quad w=(-1.04 \pm 0.04)$ & 585.42 & 0.92 & 0.924 \\
\hline $\begin{array}{c}w z C D M \text { numerical } \\
\text { integration }\end{array}$ & 639 & 4 & $H_{0}=(70 \pm 0.32) ; \quad \Omega_{\mathrm{M}}=(0.3 \pm 0.011) ; \quad w_{0}=(-1.05 \pm 0.033) ; \quad w_{1}=(0.1 \pm 0.01) ;$ & 585.59 & 0.922 & 0.92 \\
\hline Cardassian & 639 & 3 & $H_{0}=(70.10 \pm 0.42) ; \quad \Omega_{\mathrm{M}}=(0.299 \pm 0.019) ; \quad n=(-0.063 \pm 0.0095)$ & 585.43 & 0.92 & 0.924 \\
\hline
\end{tabular}




\section{Conclusions}

\section{Constant equation of state}

In the case of wCDM cosmology, we found a new analytical expression for the Hubble distance in terms of the hypergeometric function, see Equation (13). As a consequence an analytical expression for the luminosity distance and the distance modulus is derived. Two approximate Taylor expansions for the Hubble distance about $z=0$ and $z=1$ of order 7 are also derived. The derivation of the value of $w, \Omega_{\mathrm{M}}$ and $H_{0}$, here considered as a parameter to be found, is given for the Union 2.1 compilation, the JLA compilation and the Union 2.1 compilation plus the "Hymnium" GRBs sample, see Tables 1-3. As an example, in the case of the Union 2.1 compilation, we have derived $H_{0}=(70.02 \pm 0.35), \Omega_{\mathrm{M}}=(0.277 \pm 0.025)$ and $w=(-1.003 \pm 0.05)$.

\section{Variable equation of state}

In the case of $w z C D M$ cosmology the Hubble distance, Equation (23) is evaluated numerically and with a Taylor expansion of order 7, see Equation (24). The four parameters $w_{0}, w_{1}, \Omega_{\mathrm{M}}$ and $H_{0}$ are reported in Tables 1-3. As an example, in the case of the Union 2.1 compilation, we have found

$H_{0}=(70.08 \pm 0.31), \Omega_{\mathrm{M}}=(0.284 \pm 0.01), w_{0}=(-1.03 \pm 0.031)$, and $w_{1}=(0.1 \pm 0.018)$.

\section{High redshift}

The inclusion of the "Hymnium" GRBs sample allows to extend the calibration of the distance modulus up to $z=8$ (see Table 3). As an example, the Union 2.1 compilation + the "Hymnium" GRBs sample gives $H_{0}=(70 \pm 0.32), \Omega_{\mathrm{M}}=(0.3 \pm 0.011), w_{0}=(-1.05 \pm 0.033)$, and $w_{1}=(0.1 \pm 0.01)$.

\section{Cardassian cosmology}

A new solution for the Hubble radius for Cardassian cosmology is presented in terms of the hypergeometric function, see Equation (reficardz). As an example, in the case of the Union 2.1 compilation, we have derived $H_{0}=(70.15 \pm 0.38), \Omega_{\mathrm{M}}=(0.305 \pm 0.019)$ and $n=(-0.081 \pm 0.01)$.

\section{Conflicts of Interest}

The author declares no conflicts of interest regarding the publication of this paper.

\section{References}

[1] Huterer, D. and Turner, M.S. (1999) Prospects for Probing the Dark Energy via Supernova Distance Measurements. Physical Review D, 60, Article ID: 081301. https://doi.org/10.1103/PhysRevD.60.081301

[2] Huterer, D. and Shafer, D.L. (2018) Dark Energy Two Decades after: Observables, Probes, Consistency Tests. Reports on Progress in Physics, 81, Article ID: 016901. https://doi.org/10.1088/1361-6633/aa997e

[3] Brax, P. (2018) What Makes the Universe Accelerate? A Review on What Dark Energy Could Be and How to Test It. Reports on Progress in Physics, 81, Article ID 
016902. https://doi.org/10.1088/1361-6633/aa8e64

[4] Freese, K. and Lewis, M. (2002) Cardassian Expansion: A Model in Which the Universe Is Flat, Matter Dominated, and Accelerating. Physics Letters B, 540, 1-8. https://doi.org/10.1016/S0370-2693(02)02122-6

[5] Magaña, J., Amante, M.H., Garcia-Aspeitia, M.A. and Motta, V. (2018) The Cardassian Expansion Revisited: Constraints from Updated Hubble Parameter Measurements and Type Ia Supernova Data. MNRAS, 476, 1036-1049. https://doi.org/10.1093/mnras/sty260

[6] Perlmutter, S., Gabi, S., Goldhaber, G., Goobar, A., Groom, D.E., Hook, I.M., Kim, A.G., Kim, M.Y., Lee, J.C., Pain, R., Pennypacker, C.R., Small, I.A., Ellis, R.S., McMahon, R.G., Boyle, B.J., Bunclark, P.S., Carter, D., Irwin, M.J., Glazebrook, K., Newberg, H.J.M., Filippenko, A.V., Matheson, T., Dopita, M. and Couch, W.J. (1997) Measurements of the Cosmological Parameters $\Omega$ and $\Lambda$ from the First Seven Supernovae at $\mathrm{z}>0.35$. The Astrophysical Journal, 483, 565-581.

[7] Riess, A.G., Filippenko, A.V., Challis, P. and Clocchiatti, A. (1998) Observational Evidence from Supernovae for an Accelerating Universe and a Cosmological Constant. The Astronomical Journal, 116, 1009-1038. https://doi.org/10.1086/300499

[8] Perlmutter, S., Aldering, G., Goldhaber, G. and Knop, R.A. (1999) Measurements of Omega and Lambda from 42 High-Redshift Supernovae. The Astrophysical Journal, 517, 565-586. https://doi.org/10.1086/307221

[9] Suzuki, N., Rubin, D., Lidman, C., Aldering, G., Amanullah, R., Barbary, K. and Barrientos, L.F. (2012) The Hubble Space Telescope Cluster Supernova Survey. V. Improving the Dark-Energy Constraints above $\mathrm{z}$ Greater than 1 and Building an Early-Type-Hosted Supernova Sample. The Astrophysical Journal, 746, 85.

[10] Betoule, M., Kessler, R., Guy, J. and Mosher, J. (2014) Improved Cosmological Constraints from a Joint Analysis of the SDSS-II and SNLS Supernova Samples. $A s$ tronomy \& Astrophysics, 568, A22.

[11] Wei, H. (2010) Observational Constraints on Cosmological Models with the Updated Long Gamma-Ray Bursts. Journal of Cosmology and Astroparticle Physic, 8 , 020. https://doi.org/10.1088/1475-7516/2010/08/020

[12] Peebles, P.J.E. (1993) Principles of Physical Cosmology. Princeton University Press, Princeton.

[13] Tripathi, A., Sangwan, A. and Jassal, H.K. (2017) Dark Energy Equation of State Parameter and Its Evolution at Low Redshift. Journal of Cosmology and Astroparticle Physic, 6, 012. https://doi.org/10.1088/1475-7516/2017/06/012

[14] Wei, J.J., Ma, Q.B. and Wu, X.F. (2015) Utilizing the Updated Gamma-Ray Bursts and Type Ia Supernovae to Constrain the Cardassian Expansion Model and Dark Energy. Advances in Astronomy, 2015, Article ID: 576093. https://doi.org/10.1155/2015/576093

[15] Wickramasinghe, T. and Ukwatta, T.N. (2010) An Analytical Approach for the Determination of the Luminosity Distance in a Flat Universe with Dark Energy. MNRAS, 406, 548-550. https://doi.org/10.1111/j.1365-2966.2010.16686.x

[16] Press, W.H., Teukolsky, S.A., Vetterling, W.T. and Flannery, B.P. (1992) Numerical Recipes in FORTRAN. The Art of Scientific Computing. Cambridge University Press, Cambridge.

[17] Liang, N. and Zhang, S.N. (2008) Cosmology-Independent Distance Moduli of 42 Gamma-Ray Bursts between Redshift of 1.44 and 6.60. Nanjing GRB Conference, Nanjing, 23-27 June 2008, 367-372. https://doi.org/10.1063/1.3027949 
[18] Lin, H.N., Li, X. and Chang, Z. (2016) Model-Independent Distance Calibration of High-Redshift Gamma-Ray Bursts and Constrain on the $\Lambda$ CDM Model. MNRAS, 455, 2131-2138. https://doi.org/10.1093/mnras/stv2471

[19] Gupta, R.P. (2019) Weighing Cosmological Models with SNe Ia and Gamma Ray Burst Redshift Data. Universe, 5, 102. https://doi.org/10.3390/universe5050102

[20] Marosi, L.A. (2019) Extended Hubble Diagram on the Basis of Gamma Ray Bursts Including the High Redshift Range of $\mathrm{z}=0.0331-8.1$. International Journal of Astronomy and Astrophysics, 9, 1-11. https://doi.org/10.4236/ijaa.2019.91001

[21] Abramowitz, M. and Stegun, I.A. (1965) Handbook of Mathematical Functions with Formulas, Graphs, and Mathematical Tables. Dover, New York.

[22] von Seggern, D. (1992) CRC Standard Curves and Surfaces. CRC, New York.

[23] Thompson, W.J. (1997) Atlas for Computing Mathematical Functions. Wiley-Interscience, New York.

[24] Gradshteyn, I.S., Ryzhik, I.M., Jeffrey, A. and Zwillinger, D. (2007) Table of Integrals, Series, and Products. Academic Press, New York.

[25] Olver, F.W.J., Lozier, D.W., Boisvert, R.F. and Clark, C.W. (2010) NIST Handbook of Mathematical Functions. Cambridge University Press, Cambridge.

[26] Oldham, K.B., Myland, J. and Spanier, J. (2010) An Atlas of Functions: With Equator, the Atlas Function Calculator. Springer Science \& Business Media, Berlin. https://doi.org/10.1007/978-0-387-48807-3 


\section{Appendix}

\section{A. Taylor Expansion When W Is Constant}

The coefficients of the Taylor expansion of $\operatorname{Iz}_{I, 7}\left(z ; \Omega_{\mathrm{M}}, w\right)$ about $z=0$

$$
\begin{aligned}
& c_{I, 1}=1 \text {, } \\
& c_{I, 2}=3 / 4 w \Omega_{\mathrm{M}}-3 / 4 w-3 / 4, \\
& c_{I, 3}=-3 / 2 \Omega_{\mathrm{M}} w^{2}-w \Omega_{\mathrm{M}}+3 / 8 w^{2}+w+5 / 8+\frac{9 \Omega_{\mathrm{M}}^{2} w^{2}}{8}, \\
& c_{I, 4}=-\frac{71 w}{64}-\frac{9 w^{3}}{64}-\frac{35}{64}-\frac{45 w^{2}}{64}+\frac{45 \Omega_{\mathrm{M}} w^{2}}{16}-\frac{135 \Omega_{\mathrm{M}}^{2} w^{2}}{64} \\
& -\frac{243 \Omega_{\mathrm{M}}^{2} w^{3}}{64}+\frac{117 \Omega_{\mathrm{M}} w^{3}}{64}+\frac{135 \Omega_{\mathrm{M}}^{3} w^{3}}{64}+\frac{71 w \Omega_{\mathrm{M}}}{64}, \\
& c_{I, 5}=\frac{93 w}{80}+\frac{63}{128}+\frac{27 w^{3}}{80}+\frac{27 w^{4}}{640}+\frac{309 w^{2}}{320}-\frac{309 \Omega_{\mathrm{M}} w^{2}}{80}+\frac{927 \Omega_{\mathrm{M}}^{2} w^{2}}{320} \\
& +\frac{729 \Omega_{\mathrm{M}}^{2} w^{3}}{80}-\frac{351 \Omega_{\mathrm{M}} w^{3}}{80}-\frac{81 \Omega_{\mathrm{M}}^{3} w^{3}}{16}+\frac{2349 \Omega_{\mathrm{M}}^{2} w^{4}}{320}-\frac{27 \Omega_{\mathrm{M}} w^{4}}{16} \\
& -\frac{81 \Omega_{\mathrm{M}}^{3} w^{4}}{8}+\frac{567 \Omega_{\mathrm{M}}^{4} w^{4}}{128}-\frac{93 w \Omega_{\mathrm{M}}}{80}, \\
& c_{I, 6}=-\frac{3043 w}{2560}-\frac{231}{512}-\frac{27 w^{5}}{2560}-\frac{141 w^{3}}{256}-\frac{63 w^{4}}{512}-\frac{14175 \Omega_{\mathrm{M}}^{4} w^{5}}{512} \\
& +\frac{5103 \Omega_{\mathrm{M}}^{5} w^{5}}{512}-\frac{301 w^{2}}{256}+\frac{301 \Omega_{\mathrm{M}} w^{2}}{64}-\frac{903 \Omega_{\mathrm{M}}^{2} w^{2}}{256} \\
& -\frac{3807 \Omega_{\mathrm{M}}^{2} w^{3}}{256}+\frac{1833 \Omega_{\mathrm{M}} w^{3}}{256}+\frac{2115 \Omega_{\mathrm{M}}^{3} w^{3}}{256}-\frac{5481 \Omega_{\mathrm{M}}^{2} w^{4}}{256} \\
& +\frac{315 \Omega_{\mathrm{M}} w^{4}}{64}+\frac{945 \Omega_{\mathrm{M}}^{3} w^{4}}{32}-\frac{6615 \Omega_{\mathrm{M}}^{4} w^{4}}{512}-\frac{2673 \Omega_{\mathrm{M}}^{2} w^{5}}{256} \\
& +\frac{3267 \Omega_{\mathrm{M}} w^{5}}{2560}+\frac{6885 \Omega_{\mathrm{M}}^{3} w^{5}}{256}+\frac{3043 w \Omega_{\mathrm{M}}}{2560}, \\
& c_{I, 7}=\frac{2689 w}{2240}+\frac{81 w^{6}}{35840}+\frac{81 w^{5}}{2240}+\frac{171 w^{3}}{224}+\frac{1665 w^{4}}{7168}+\frac{48259 w^{2}}{35840}+\frac{429}{1024} \\
& +\frac{95985 \Omega_{\mathrm{M}}^{4} w^{6}}{1024}-\frac{19683 \Omega_{\mathrm{M}}^{5} w^{6}}{256}+\frac{24057 \Omega_{\mathrm{M}}^{6} w^{6}}{1024}+\frac{61479 \Omega_{\mathrm{M}}^{2} w^{6}}{5120} \\
& -\frac{1053 \Omega_{\mathrm{M}} w^{6}}{1280}-\frac{23085 \Omega_{\mathrm{M}}^{3} w^{6}}{448}+\frac{6075 \Omega_{\mathrm{M}}^{4} w^{5}}{64}-\frac{2187 \Omega_{\mathrm{M}}^{5} w^{5}}{64} \\
& +\frac{8019 \Omega_{\mathrm{M}}^{2} w^{5}}{224}-\frac{9801 \Omega_{\mathrm{M}} w^{5}}{2240}-\frac{20655 \Omega_{\mathrm{M}}^{3} w^{5}}{224}+\frac{144855 \Omega_{\mathrm{M}}^{2} w^{4}}{3584} \\
& -\frac{8325 \Omega_{\mathrm{M}} w^{4}}{896}-\frac{24975 \Omega_{\mathrm{M}}^{3} w^{4}}{448}+\frac{24975 \Omega_{\mathrm{M}}^{4} w^{4}}{1024}+\frac{4617 \Omega_{\mathrm{M}}^{2} w^{3}}{224} \\
& -\frac{2223 \Omega_{\mathrm{M}} w^{3}}{224}-\frac{2565 \Omega_{\mathrm{M}}^{3} w^{3}}{224}-\frac{48259 \Omega_{\mathrm{M}} w^{2}}{8960}+\frac{144777 \Omega_{\mathrm{M}}^{2} w^{2}}{35840} \\
& -\frac{2689 w \Omega_{\mathrm{M}}}{2240} \text {. }
\end{aligned}
$$

The integral of the Taylor expansion of order 2 about $z=1$ is 


$$
I z_{I I, 2}=\frac{N}{D}
$$

where

$$
\begin{aligned}
N= & \left(3 \times 8^{w} \Omega_{\mathrm{M}} w z-6 \times 8^{w} w \Omega_{\mathrm{M}}+3 \times 8^{w} \Omega_{\mathrm{M}} z-3 w z 8^{w}-14 \times 8^{w} \Omega_{\mathrm{M}}\right. \\
& \left.+6 w 8^{w}-3 z 8^{w}-3 \Omega_{\mathrm{M}} z+14 \times 8^{w}+14 \Omega_{\mathrm{M}}\right) z
\end{aligned}
$$

and

$$
D=\left(-2^{3+3 w} \Omega_{\mathrm{M}}+2^{3+3 w}+8 \Omega_{\mathrm{M}}\right)^{3 / 2} .
$$

\section{B. The Hypergeometric Function}

The regularized hypergeometric function, ${ }_{2} \mathrm{~F}_{1}(a, b ; c ; z)$, as defined by the Gauss series, is

$$
\begin{aligned}
{ }_{2} \mathrm{~F}_{1}(a, b ; c ; z) & =\sum_{s=0}^{\infty} \frac{(a)_{s}(b)_{s}}{(c)_{s} s !} z^{s}=1+\frac{a b}{c} z+\frac{a(a+1) b(b+1)}{c(c+1) 2 !} z^{2}+\cdots \\
& =\frac{\Gamma(c)}{\Gamma(a) \Gamma(b)} \sum_{s=0}^{\infty} \frac{\Gamma(a+s) \Gamma(b+s)}{\Gamma(c+s) s !} z^{s}
\end{aligned}
$$

where $z=x+i y,(a)_{s}$ is the Pochhammer symbol

$$
(a)_{s}=a(a+1) \cdots(a+s-1),
$$

$\Gamma(z)$ is the Gamma function defined as

$$
\Gamma(z)=\int_{0}^{\infty} \mathrm{e}^{-t} t^{z-1} \mathrm{~d} t
$$

$Z$ is a complex variable defined on the disk $|z|<1$ that should not be confused with the redshift, see [21] [22] [23] [24] [25]. The following relationship

$$
{ }_{2} \mathrm{~F}_{1}(a, b ; c ; x)=(1-x)^{-a}{ }_{2} \mathrm{~F}_{1}\left(a, c-b ; c ; \frac{x}{x-1}\right)
$$

connect the the hypergeometric function with $x$ in $(-1,1)$ to one with $x$ in $\left(-\infty, \frac{1}{2}\right)$, see more details in [26].

\section{Taylor Expansion When W Is Variable}

The coefficients of the Taylor expansion of $\operatorname{Iwz}_{I, 7}\left(z ; \Omega_{\mathrm{M}}, w_{0}, w_{1}\right)$ about $z=0$

$$
\begin{gathered}
c_{I, 1}=1, \\
c_{I, 2}=\frac{3}{4} w_{0} \Omega_{\mathrm{M}}-\frac{3}{4} w_{0}-\frac{3}{4}, \\
c_{I, 3}=5 / 8+w_{0}-1 / 4 w_{1}+1 / 4 w_{1} \Omega_{\mathrm{M}}-w_{0} \Omega_{\mathrm{M}}+3 / 8 w_{0}^{2}-3 / 2 \Omega_{\mathrm{M}} w_{0}^{2}+\frac{9 \Omega_{\mathrm{M}}^{2} w_{0}^{2}}{8}, \\
c_{I, 4}=-\frac{35}{64}-\frac{71 w_{0}}{64}+\frac{17 w_{1}}{32}-\frac{17 w_{1} \Omega_{\mathrm{M}}}{32}+\frac{71 w_{0} \Omega_{\mathrm{M}}}{64}-\frac{45 w_{0}^{2}}{64}+\frac{9 w_{0} w_{1}}{32} \\
+\frac{45 \Omega_{\mathrm{M}} w_{0}^{2}}{16}-\frac{135 \Omega_{\mathrm{M}}^{2} w_{0}^{2}}{64}-\frac{243 \Omega_{\mathrm{M}}^{2} w_{0}^{3}}{64}+\frac{117 \Omega_{\mathrm{M}} w_{0}^{3}}{64} \\
+\frac{135 \Omega_{\mathrm{M}}^{3} w_{0}^{3}}{64}-\frac{9 w_{0}^{3}}{64}-\frac{9 \Omega_{\mathrm{M}} w_{0} w_{1}}{8}+\frac{27 \Omega_{\mathrm{M}}^{2} w_{0} w_{1}}{32},
\end{gathered}
$$




$$
\begin{aligned}
c_{I, 5}= & \frac{27 w_{0}^{3}}{80}+\frac{63}{128}-\frac{9 w_{1}^{2} \Omega_{\mathrm{M}}}{40}+\frac{2349 \Omega_{\mathrm{M}}^{2} w_{0}^{4}}{320}-\frac{27 \Omega_{\mathrm{M}} w_{0}^{4}}{16}+\frac{27 w_{1}^{2} \Omega_{\mathrm{M}}^{2}}{160} \\
& -\frac{81 \Omega_{\mathrm{M}}^{3} w_{0}^{4}}{8}+\frac{567 \Omega_{\mathrm{M}}^{4} w_{0}^{3}}{128}-\frac{27 w_{0}^{2} w_{1}}{160}+\frac{309 w_{0}^{2}}{320}-\frac{3}{4} w_{0} w_{1}+\frac{729 \Omega_{\mathrm{M}}^{2} w_{0}^{3}}{80} \\
& -\frac{351 \Omega_{\mathrm{M}} w_{0}^{3}}{80}-\frac{81 \Omega_{\mathrm{M}}^{3} w_{0}^{3}}{16}+\frac{93 w_{0}}{80}-\frac{129 w_{1}}{160}+\frac{9 w_{1}^{2}}{160}+\frac{27 w_{0}^{4}}{640} \\
& +\frac{351 \Omega_{\mathrm{M}} w_{0}^{2} w_{1}}{160}+\frac{129 w_{1} \Omega_{\mathrm{M}}}{160}-\frac{309 \Omega_{\mathrm{M}} w_{0}^{2}}{80}+\frac{927 \Omega_{\mathrm{M}}^{2} w_{0}^{2}}{320}-\frac{93 w_{0} \Omega_{\mathrm{M}}}{80} \\
& +\frac{81 \Omega_{\mathrm{M}}^{3} w_{0}^{2} w_{1}}{32}-\frac{729 \Omega_{\mathrm{M}}^{2} w_{0}^{2} w_{1}}{160}-\frac{9}{4} \Omega_{\mathrm{M}}^{2} w_{0} w_{1}+3 \Omega_{\mathrm{M}} w_{0} w_{1} .
\end{aligned}
$$

The integral of the Taylor expansion of order 2 about $z=1$ in the case wzLCDM cosmology

$$
I w z_{I I, 2}=\frac{N w z}{D w z},
$$

where

$$
\begin{aligned}
N w z= & \mathrm{e}^{\frac{3}{4} w_{1}}\left(6 \times 2^{1 / 2+3 w_{0}+3 w_{1}} \Omega_{\mathrm{M}} z w_{0}+3 \times 2^{1 / 2+3 w_{0}+3 w_{1}} \Omega_{\mathrm{M}} z w_{1}-6 \mathrm{e}^{3 / 2 w_{1}} \Omega_{\mathrm{M}} \sqrt{2} z\right. \\
& +6 \times 2^{1 / 2+3 w_{0}+3 w_{1}} \Omega_{\mathrm{M}} z-12 \times 2^{1 / 2+3 w_{0}+3 w_{1}} w_{0} \Omega_{\mathrm{M}}-6 \times 2^{1 / 2+3 w_{0}+3 w_{1}} \Omega_{\mathrm{M}} w_{1} \\
& -6 \times 2^{1 / 2+3 w_{0}+3 w_{1}} z w_{0}-3 \times 2^{1 / 2+3 w_{0}+3 w_{1}} z w_{1}+28 \mathrm{e}^{3 / 2 w_{1}} \Omega_{\mathrm{M}} \sqrt{2} \\
& -28 \times 2^{1 / 2+3 w_{0}+3 w_{1}} \Omega_{\mathrm{M}}-6 \times 2^{1 / 2+3 w_{0}+3 w_{1}} z+12 \times 2^{1 / 2+3 w_{0}+3 w_{1}} w_{0} \\
& \left.+6 \times 2^{1 / 2+3 w_{0}+3 w_{1}} w_{1}+28 \times 2^{1 / 2+3 w_{0}+3 w_{1}}\right) z
\end{aligned}
$$

and

$$
D w z=64\left(-\Omega_{\mathrm{M}} 2^{3 w_{0}+3 w_{1}}+2^{3 w_{0}+3 w_{1}}+\Omega_{\mathrm{M}} \mathrm{e}^{3 / 2 w_{1}}\right)^{3 / 2} .
$$

